\title{
Supporting Information for \\ Self-Supported Organometallic Rhodium Quinonoid Nanocatalysts \\ for Stereoselective Polymerization of Phenylacethylene
}

Kang Hyun Park, ${ }^{\dagger}$ Kwon Ho Jang, ${ }^{\dagger}$ Seung Uk Son, ${ }^{*}{ }^{\dagger}$ and Dwight A. Sweigart*,,

Department of Chemistry, Sungkyunkwan University, Suwon 440-746, Korea, and

Department of Chemisrty, Brown University, Providence, RI 02912, USA

\section{Experimental}

Characterization of Organometallic Nanocatalysts: The sizes measurement and energy dispersive spectroscopy (EDS) were preformed using a FE-SEM (JSM6700F). ${ }^{27} \mathrm{Al}$ MAS NMR spectra were recorded on a Bruker $400 \mathrm{MHz}$ Solid State Bruker DSX NMR spectrometer at Korea Basic Science Institute (Daegu) operating at 104.3 MHz Larmor frequency. The spinning speed of $13.5 \mathrm{kHz}$ was employed. The repetition delay time was 10 sec. All spectra were collected by using a single pulse sequence. ${ }^{27} \mathrm{Al}$ spectrum of organometallic nanocatalyst was calibrated to aluminum chloride $\left(\mathrm{AlCl}_{3}\right)$ at $0 \mathrm{ppm}$. X-ray photoelectron spectroscopy (XPS) was obtained using a Thermo VG and Monochromatic Al- $K \alpha$ radiation. ICP-atomic emission spectroscopy(AES) experiment was performed by Shimadzu ICPS-1000 $\square$.

Characterization of Polymers: Room temperature absorption and emission spectra of poly(phenylacetylene)s were recorded using Jasco V-500 and Jasco FP-6500 spectrophotometers, respectively. For UV and PL experiments, $\mathrm{CH}_{2} \mathrm{Cl}_{2}$ (HPLC grade, Burdick \& Jackson, 50mL) solutions of $4 \mathrm{mg}$ of poly(phenylacetylene) were used. The molecular weights and molecular weight distributions of the polymers were determined by gel permeation chromatography (GPC) utilizing a Waters model 1515 pump and a model 2414 refractive index detector with three Styragel columns HR 5E (pore size: linear type

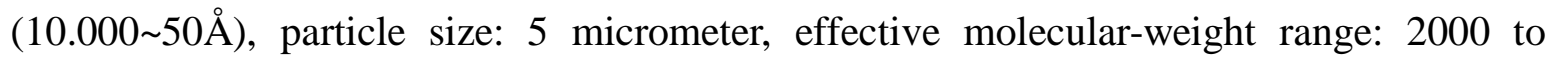
4,000,000), HR 5E (pore size: linear type (10.000 50 $)$ ), particle size: 5 micrometer, effective molecular-weight range: 2000 to 4,000,000), HR 2 (pore size: 500A, particle size: 5 micrometer, effective molecular-weight range: 500 to 20,000) connected in a serial fashion. $\mathrm{CHCl}_{3}$ was used as the eluent at a flow rate of $1.0 \mathrm{~mL} / \mathrm{min}$ and calibrated with polystyrene standards at $35{ }^{\circ} \mathrm{C}$. 


\section{Typical Synthetic Procedure for Organometallic Nanocatalyst(ON3)}

After flame drying the glassware, $\left[\left(\mathrm{H}_{2} \mathrm{Q}\right) \mathrm{Rh}(\mathrm{COD})\right] \mathrm{BF}_{4}(0.10 \mathrm{~g}, 0.25 \mathrm{mmol})$ in $20 \mathrm{~mL} \mathrm{THF}$ and $\mathrm{Al}\left(\mathrm{O}^{i} \mathrm{Pr}\right)_{3}(50.04 \mathrm{mg}, 0.25 \mathrm{mmol})$ in $3 \mathrm{~mL}$ THF were mixed for $2 \mathrm{~h}$ at room temperature. During the reaction, the yellow precipitates were formed. They were retrieved by centrifugation and washed 3 times using $20 \mathrm{~mL}$ THF and dried in vacuo. The isolated yield was $87 \%$ based on the mole number of rhodium atom used.

\section{Typical Procedure of Polymerization of Phenylacethylene}

$19 \mathrm{mg}$ (5.7mg of $\mathrm{Rh}, 1.2 \mathrm{~mol} \%$ ) of organometallic nanocatalyst was added to $10 \mathrm{~mL}$ Schlenk tube dried by flame under argon. Via a glass syringe, dry THF $(2.5 \mathrm{~mL})$ and phenylacethylene $(0.5 \mathrm{ml}, 4.55 \mathrm{mmol})$ were added under argon. After stirred for $18 \mathrm{~h}$, the reaction mixture was centrifuged to recover the yellow organometallic nanocatalyst. The methanol $(20 \mathrm{~mL})$ was added to the clear solution to form the precipitates of polymer, which were retrieved by centrifugation and washed 3 times by excess methanol $(20 \mathrm{~mL} \times 3)$ and dried under vacuum.

S1. Size distributions of synthesized nanocatalysts.

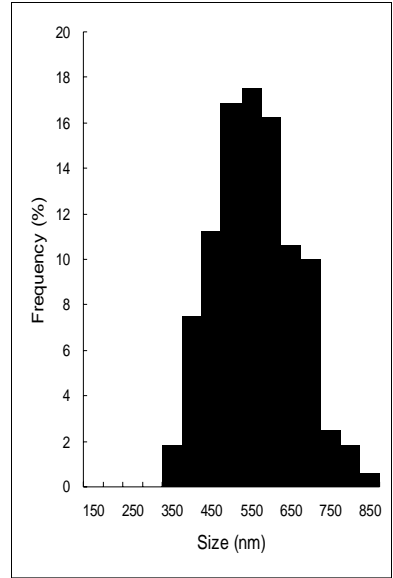

ON1

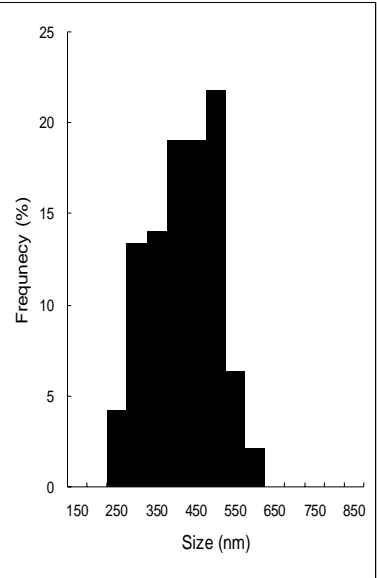

ON2

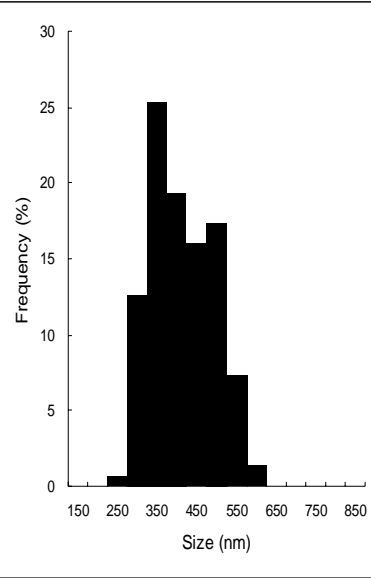

ON2-Al

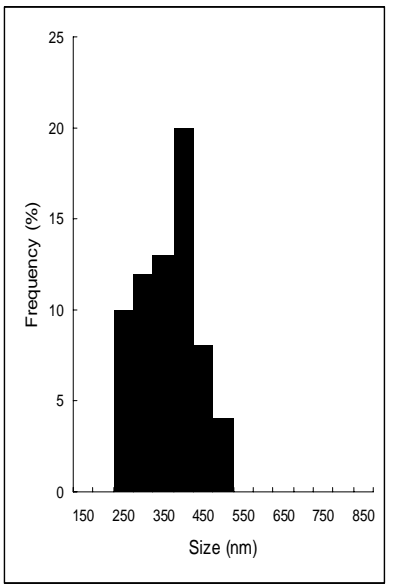

ON3

\begin{tabular}{|l|c|c|c|c|}
\hline Organometallic Catalysts & ON1 & ON2 & ON2-Al & ON3 \\
\hline Mean Sizes(nm) & 537 & 402 & 399 & 340 \\
\hline Standard Deviation (\%) & 19 & 23 & 21 & 21 \\
\hline
\end{tabular}


S2. SEM images of ON3, before (a) and after use (b).

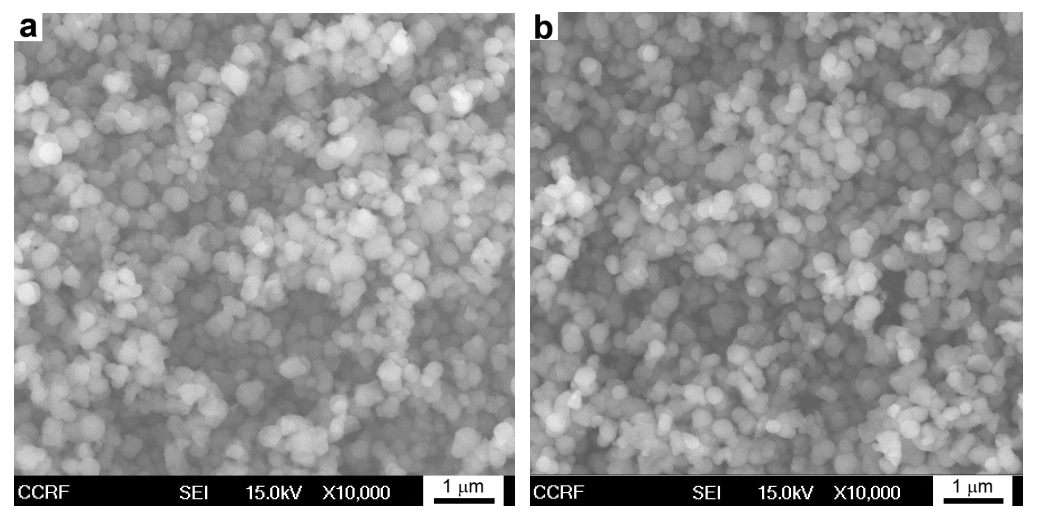

S3. ${ }^{1} \mathrm{H}$ NMR spectra of synthesized polymers in table 1 .

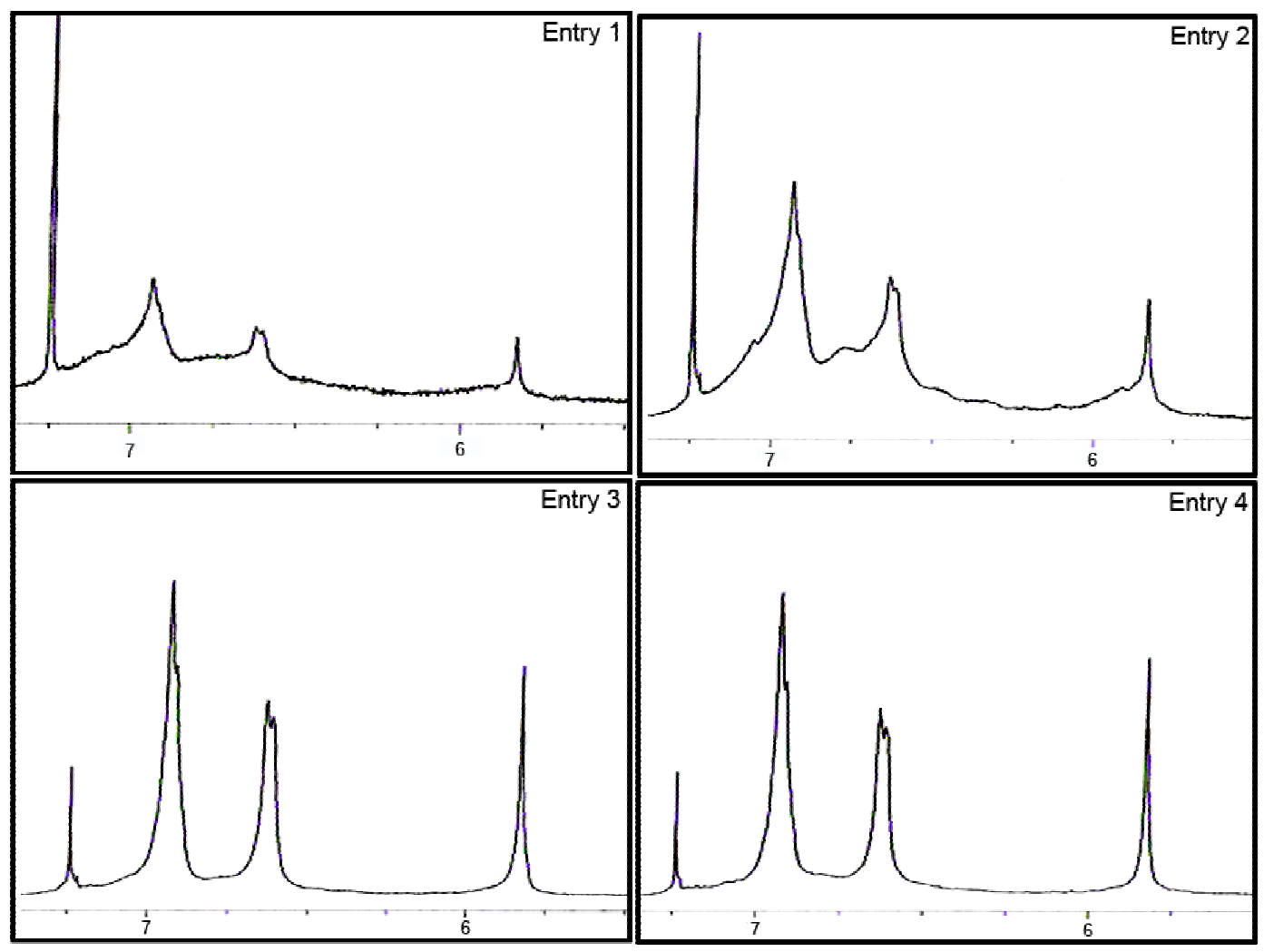




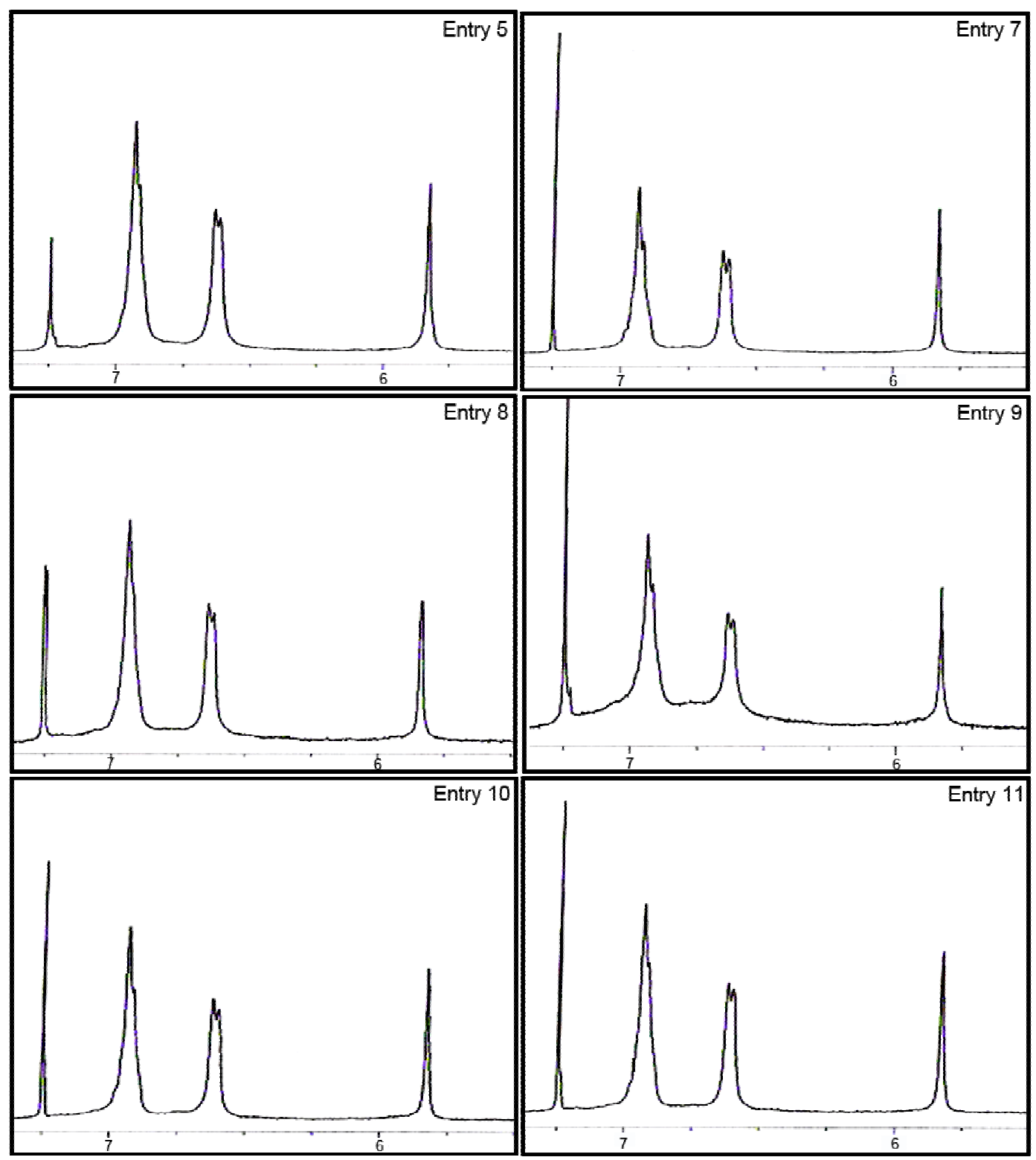

S4. XPS spectrum of Al 2p orbital in organometallic nanocatalyst.

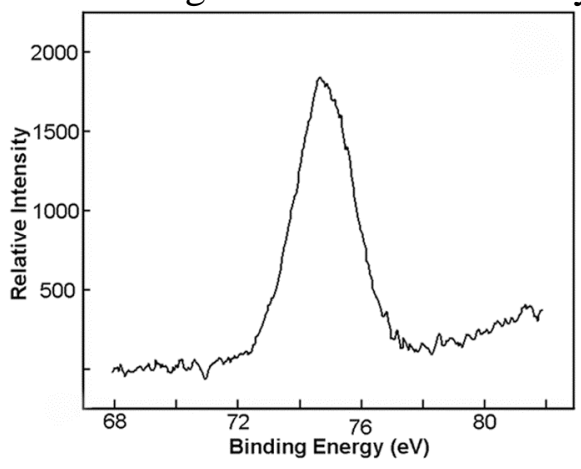

